\title{
Thoracoscopic repair of cervical lung hernia
}

Sanghoon Jheon, MD, Eung Bae Lee, MD, Joon Yong Cho, MD, Bong Hyun Chang, MD, Jongtae Lee, MD, and

Kyu Tae Kim, MD, Daegu, Korea

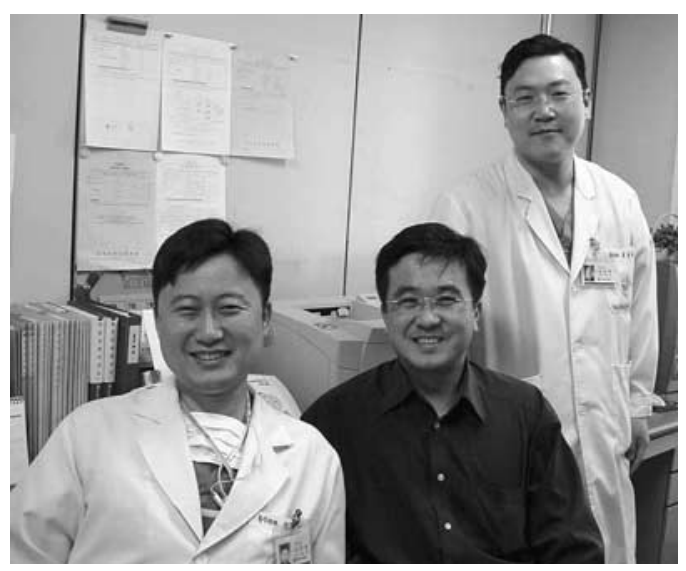

Cho, Jheon, and Gong (left to right)

$\mathrm{T}$ he rare condition of lung hernia is abnormal protrusion of the lung beyond the confines of thoracic cage through an abnormal opening in the chest wall. It is classified as cervical, thoracic, or diaphragmatic according to the location and as congenital or acquired according to the etiology. ${ }^{1}$ The most common form is the acquired thoracic type as a result of trauma or surgical incision. Cervical lung hernia in an adult is rare, and surgical management for patients with symptoms has seldom been reported. We managed this condition with thoracoscopic surgery with excellent long-term result. To our knowledge, thora-

From the Department of Thoracic and Cardiovascular Surgery, School of Medicine, Kyungpook National University, Daegu, Korea.

S.J. is currently at Department of Thoracic and Cardiovascular Surgery, School of Medicine, Catholic University of Daegu, Korea.

Received for publication April 11, 2002; accepted for publication April 22, 2002 .

Address for reprints: Sanghoon Jheon, MD, Department of Thoracic and Cardiovascular Surgery, School of Medicine, Catholic University of Daegu, 3056-6, Daemyung 4-dong, Namgu, Daegu 705-718, Korea (E-mail: jheon@cataegu.ac.kr).

J Thorac Cardiovasc Surg 2002;124:1030-1

Copyright (C) 2002 by The American Association for Thoracic Surgery

$0022-5223 / 2002 \$ 35.00+0 \quad \mathbf{1 2 / 5 4 / 1 2 5 6 8 9}$

doi: $10.1067 / \mathrm{mtc} .2002 .125689$ coscopic management for cervical lung hernia has not previously been reported.

\section{Clinical Summary}

A 41-year-old obese man visited the hospital with neck discomfort and a right cervical mass. The patient reported cervical pain after exercise, dyspnea on exertion, and severe snoring with a particularly deep, resonant voice. Physical examination revealed about a $10-\mathrm{cm}$ mass in the right supraclavicular area. This mass increased in size with coughing or the Valsalva maneuver. It was easily reducible into the infraclavicular area, and breathing sounds could easily be heard over the mass. A chest radiograph showed tracheal deviation and a large air shadow on the right supraclavicular area reaching to the submental area (Figure 1).

The procedure was done with three thoracoports, a doublelumen endotracheal tube, and general anesthesia. The herniated portion of the lung revealed severe emphysematous change and was resected with an endoscopic stapling device. The thickened hernia sac was everted and allowed to retract into the thoracic cavity. Fatty tissue around the sac was removed, and excess hernia sac was trimmed away. The endoscopic screw and hernia stapler were used to fix the remaining hernia sac to the second rib to close the hernia defect. Reinforcing sutures were added to the sac around the intercostal muscle. The postoperative course was uneventful, and the patient remains free of symptoms and without evidence of recurrence 5 years later (Figure 2). 


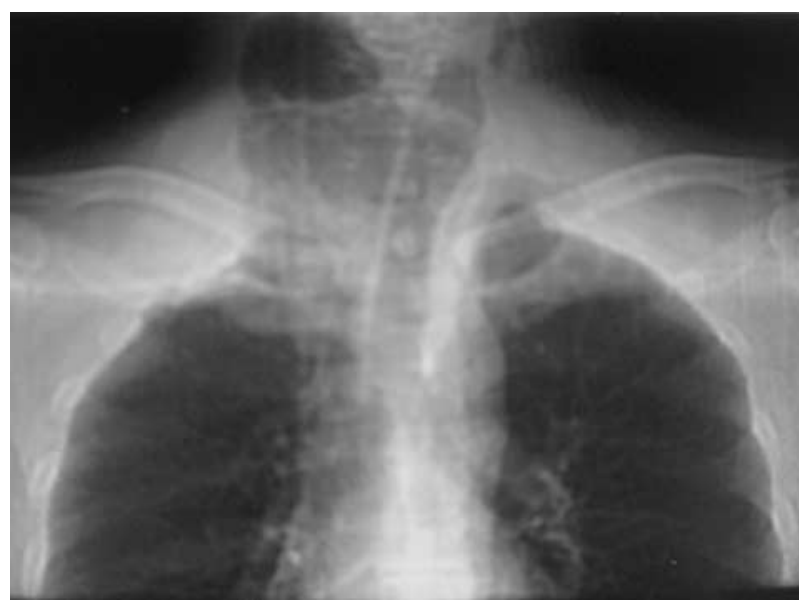

Figure 1. Chest radiograph shows large apical radiolucency extending to the base of the neck.

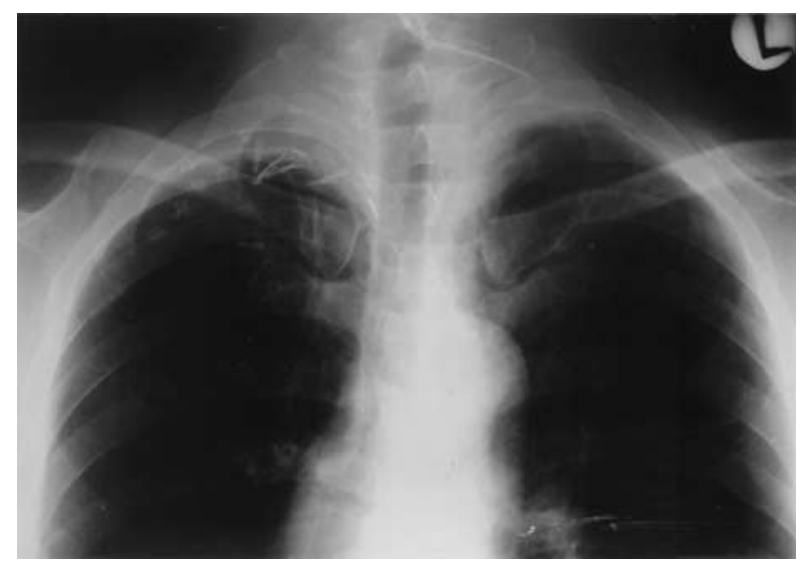

Figure 2. Postoperative chest radiograph shows normal configuration of the right lung apex.

\section{Discussion}

Most congenital cervical lung hernias in the pediatric age group tend to resolve spontaneously. ${ }^{2}$ In adults, however, this may rather represent an acquired condition. In fact, it has been reported in wind instrumentalists, patients with chronic cough, patients with emphysema, weight lifters, and patients with a history of prolonged or repeated Valsalva maneuver. ${ }^{3}$

Anatomically, the thoracic outlet is covered by parietal pleura and Sibson fascia, which prevent the apex of the lung from bulging out to the neck. Sibson fascia is an extension of endothoracic fascia from the transverse process of the first thoracic vertebra to anterolateral margin of the first rib. When it is weakened or torn, a cervical hernia will occur through weak area of anteromedial side.

Most reported cases have been asymptomatic. However, this condition may cause cough or dysphagia from compression, cervical pain suggestive of incarceration, and the cosmetic problem of supraclavicular bulging. Patients usually have resonant a voice and severe snoring because the air chamber is near the vocal cord.

In chest radiographs, cervical lung hernias manifest as apical radiolucencies in varying sizes extending to the base of the neck. Usually the hernia causes lateral deviation of the trachea. Maximal size of hernia may be observed with the Valsalva maneuver.

Management is usually conservative rather than surgical. Surgical indications include symptoms such as airway obstruction signs, incarceration, cosmetic deformities, interference with usual activity, or recurrent infection.

Formerly, cervical ${ }^{4}$ or thoracic 5 approaches were performed. The cervical approach provide excellent exposure but potentially higher risk of recurrence, because closure of the defect is more difficult than with the transthoracic approach. The conventional thoracic approach is more invasive method, however, with a large incision.

Because of advances in video technology and endoscopic surgical instruments, we tried a thoracoscopic approach. This is the first such trial, according to our English-language literature review. Herniated portions of the lung usually become somewhat emphysematous because of air trapping and thus require wedge resection with endoscopic stapling device. The hernia sac should be reduced and properly fixed after trimming out to close the defect. Fat tissue around hernia sac should be removed, because it could enhance recurrence. We used hernia staplers and endoscrews to fix the hernia sac to the chest wall to patch the defect, but these were not satisfactory. With suturing on intercostal muscle, we could fix the sac tightly. The thoracoscopic approach provides excellent surgical view, cosmetic merit, convenience for lung resection, and a proper approach to close the defect away from the pleural cavity and thus disperse pushing power that might encourage recurrence.

\section{References}

1. Maurer E, Blades B. Hernia of the lung. J Thorac Surg. 1946;15:7798.

2. Grunebaum M, Griscom NT. Protrusion of the lung apex through Sibson's fascia in infancy. Thorax. 1978;33:290-4.

3. McAdams HP, Gordon DS, White CS. Apical lung hernia: radiologic findings in six cases. AJR Am J Roentgenol. 1996;167:927-30.

4. Victor S, Muthurajan S, Sekhar TG, Gopinath R, Dhala B, Devi V, et al. Giant cervical herniation of an apical pulmonary bulla. $J$ Thorac Cardiovasc Surg. 1987;93:141-7.

5. Lightwood RG, Cleland WP. Cervical lung hernia. Thorax. 1974;29: 349-51. 